\title{
ON THE NUMBER OF SOLUTIONS OF THE EQUATION $x^{p^{k}}=a$ IN A FINITE $p$-GROUP
}

\author{
YAKOV G. BERKOVICH \\ (Communicated by Warren J. Wong) \\ Dedicated to Professor Gregory A. Freiman
}

\begin{abstract}
A. Kulakoff (Math. Ann. 104 (1931), 778-793) proved that for $p>$ 2 the number of solutions of the equation $x^{p^{k}}=e \quad(e$ is a unit element of $G)$ in a finite noncyclic $p$-group $G$ is divisible by $p^{k+1}$ if $\exp G \geq p^{k}$. In this note we consider the number $N(a, G, k)$ of solutions of the equation $x^{p^{k}}=a$ in $G, a \in G$. Our results cover the case $p=2$ also.
\end{abstract}

\section{INTRODUCTION}

In this note $p$ denotes a prime and $G$ denotes a finite $p$-group.

A $p$-group $G$ is called regular (Hall [8]) if for all $x, y \in G$ there exists $z \in\langle x, y\rangle$ such that $(x y)^{p}=x^{p} y^{p} z^{p}$. By a well-known theorem of Hall [8] for an irregular $p$-group $G$ we have

$$
\left|G:\left\langle x^{p} \mid x \in G\right\rangle\right| \geq p^{p} .
$$

A $p$-group $G$ is called absolutely regular (Blackburn [6]) if $\left|G:\left\langle x^{p} \mid x \in G\right\rangle\right|<$ $p^{p}$. Hence an absolutely regular $p$-group is regular.

If a $p$-group $G$ is regular and $k$ is a positive integer, then

$$
N(e, G, k)=\left|\left\{x \in G \mid x^{p^{k}}=e\right\}\right|,
$$

i.e., $N(e, G, k)$ depends on the power structure of $G$ only. We note that for a regular $p$-group $G$ we have

$$
\left\langle x \in G \mid x^{p^{k}}=e\right\rangle=\left\{x \in G \mid x^{p^{k}}=e\right\},
$$

i.e., $\exp \left\langle x \in G \mid x^{p^{k}}=e\right\rangle \leq p^{k}$.

Hence $N(e, G, k)$ is hard to determine only for irregular $p$-groups.

Obviously an absolutely regular 2-group is cyclic. We note that an absolutely regular 3-group is metacyclic.

If $p>2$ and a $p$-group $G$ is noncyclic, $\exp G \geq p^{k}$, then $N(e, G, k)$ is divisible by $p^{k+1}$ (Kulakoff [10]).

We call a $p$-group $G$ exceptional if it is absolutely regular or of maximal class. Blackburn [6] showed that any nonexceptional $p$-group contains a normal

Received by the editors April 7, 1989.

1991 Mathematics Subject Classification. Primary 20D10; Secondary 20D60. 
subgroup of order $p^{p}$ and exponent $p$. If a 2-group $G$ is nonexceptional then $N(e, G, 1)$ is divisible by $4[2 ; 11$, Theorem $6 ; 9$, Theorem 4.9]. This result was generalized by Berkovich [1] and Blackburn [7]: if $G$ is a nonexceptional $p$-group then $N(e, G, 1)$ is divisible by $p^{p}$. This is the best-possible result.

If $a \neq e$ the counting of $N(a, G, k)$ is harder and considerably more subtle. If $p>2$ and a $p$-group $G$ is noncyclic then $N(a, G, k)$ is divisible by $p^{2}$ (Lam [12]). On pp. 580-581 of paper [12] Lam writes: "It seems likely that, more generally, for any central element $a \in Z(G)$ the number of solutions of $x^{2}=a$ in (nonexceptional 2-group) $G$ is divisible by 4 , but we have not been able to find a proof."

Theorem A shows that Lam's conjecture is true. This theorem shows that as a rule $N(e, G, k)$ is divisible by $p^{k+1}$ if $\exp G \geq p^{k}$. Theorem B shows that in a nonexceptional $p$-group $G$ the number $N(e, G, k)$ is divisible by $p^{k+p-1}$ if $\exp G \geq p^{k}$. Both these theorems extend results mentioned earlier.

We denote by $G^{\prime}$ the commutator subgroup of $G$ and by $\varphi(G)$ the Frattini subgroup of $G$. Since in a $p$-group we have

$$
\varphi(G)=G^{\prime}\left\langle x^{p} \mid x \in G\right\rangle,
$$

it follows that $H \leq G \Rightarrow \varphi(H) \leq \varphi(G)$. If $A$ is a subset of $G$ then $C_{G}(A)$, resp. $N_{G}(A)$, denotes the centralizer, resp. normalize, r of $A$ in $G$. By $|\mathfrak{M}|$ we denote the number of elements of a set $\mathfrak{M}$.

\section{Results}

In this section we state our main results.

Theorem A. Let a p-group $G$ be noncyclic and not a 2-group of maximal class. Let $k$ be a positive integer, $a \in G$, and $\exp G \geq p^{k}|\langle a\rangle|$. Then $N(a, G, k)$ is divisible by $p^{k+1}$.

In particular we see that Lam's conjecture holds.

Theorem B. Let a p-group $G$ be nonexceptional, $k$ be a positive integer, and $\exp G \geq p^{k}$. Then $N(e, G, k)$ is divisible by $p^{k+p-1}$.

In particular in the case when $a=e$ and $p=2$, Theorem B implies Theorem A. We note that the proof of Theorem A is completely elementary, but the proof of Theorem B uses deep results of $p$-group theory.

\section{Proofs}

In this section we prove Theorems A and $\mathrm{B}$.

Lemma 1. Let $G$ be a cyclic p-group, let $k$ be a positive integer, $a \in G$, and $|G| \geq p^{k}|\langle a\rangle|$. Then $N(a, G, k)=p^{k}$.

This is obvious.

Lemma 2. Let $G$ be a 2-group of maximal class, $k$ be a positive integer, $a \in G$. If $a=e$ then let $k>1$. Then $N(a, G, k)=0$ or $N(a, G, k) \equiv 2^{k}$ $\left(\bmod 2^{k+1}\right)$.

This is proved by easy checking since a 2-group of maximal class is dihedral, semi-dihedral, or generalized quaternion.

We note that, in Lemma 2 , if $N(a, G, k)>0$ then $a \in \varphi(G)$. 
Lemma 3. Let $A$ be a cyclic subgroup of a p-group $G, C_{G}(A)>A$. If $C_{G}(A)$ is cyclic then $G$ is cyclic or a 2-group of maximal class.

Proof. Suppose that $G$ is noncyclic and not a 2-group of maximal class. Then by Roquette's theorem (see, e.g., [3]) $G$ contains a normal subgroup $R$ of type $(p, p)$. Then $R C_{G}(A)$ is a nonabelian $p$-group with a cyclic subgroup of index $p$ and $A \not \leq Z\left(R C_{G}(A)\right)$. Since $A<C_{G}(A)$,

$$
A \leq \varphi\left(C_{G}(A)\right) \leq \varphi\left(R C_{G}(A)\right)=Z\left(R C_{G}(A)\right)
$$

(the equality follows from the classification of $p$-groups with a cyclic subgroup of index $p$ ), a contradiction.

Note that if $A$ is a cyclic subgroup of a 2-group of maximal class and $|A|>2$ then $C_{G}(A)$ is cyclic (see remark after Lemma 2).

Lemma 4. Let $A$ be a cyclic subgroup of a noncyclic p-group $G$, where $G$ is not a 2-group of maximal class. Let $k$ be a positive integer and $k>1$ if $|A|=1$. Let $\mathfrak{M}=\mathfrak{M}(A, G, k)$ denote the set of all cyclic subgroups containing $A$ of order $p^{k}|A|$ in $G$. Then $|\mathfrak{M}| \equiv 0(\bmod p)$.

Proof. For $|A|=1$ this result is well known (for $p>2$ it is due to Miller and for $p=2$ it is due to Berkovich; for details see $[1,3])$. Now let $|A|>1$.

Induct on $|G|$.

For $D \leq G$ let $c(D)$ denote the number of elements of $\mathfrak{M}$ contained in $D$ (if $A \leq D$ then $c(D)=|\mathfrak{M}(A, D, k)|$ and if $A \not \leq D$ then $c(D)=0)$.

We may assume that the set $\mathfrak{M}$ is nonempty. Obviously $\mathfrak{M}(A, G, k)=$ $\mathfrak{M}\left(A, C_{G}(A), k\right)$. Since $\mathfrak{M}$ is nonempty, $C_{G}(A)>A$ and $C_{G}(A)$ is noncyclic by Lemma 3. If $C_{G}(A)$ is a 2-group of maximal class then $|A|=2$ and elementary results of $p$-group theory imply $G=C_{G}(A)$, a contradiction to the assumption that $G$ is not a 2-group of maximal class.

If $C_{G}(A)<G$ then $|\mathfrak{M}| \equiv 0(\bmod p)$ by induction. Hence we may assume that $C_{G}(A)=G$, i.e., $A \leq Z(G)$.

Since $\mathfrak{M}$ is nonempty, for $B \in \mathfrak{M}$ we have $A \leq \varphi(B) \leq \varphi(G)$. Let $T_{1}, \ldots, T_{m}$ be all maximal subgroups of $G$. Then by Hall's enumeration principle [8] we have

$$
|\mathfrak{M}|=c(G) \equiv \sum_{i=1}^{m} c\left(T_{i}\right) \quad(\bmod p) .
$$

Suppose that one of the $T_{i}$ 's, say $T_{1}$, is cyclic. Then $m=p+1$ and exactly $p$ of the $T_{i}$ 's are cyclic (this follows from the classification of $p$-groups with a cyclic subgroup of index $p$ ). Since $A \leq \varphi(G), c\left(T_{i}\right)=1$ for all cyclic $T_{i}$. If $T_{j}$ is noncyclic (then $T_{j}$ is abelian) then $c\left(T_{j}\right)=0$ or $p$, and (1) gives $|\mathfrak{M}| \equiv 0$ $(\bmod p)$. Hence we may assume that any $T_{i}$ is noncyclic. Suppose that one of the $T_{i}$ 's, say $T_{1}$, is a 2-group of maximal class. By a result of Berkovich (see $\S 5$ in [3]) among $T_{i}$ 's there are $4 t \quad(t \geq 1$ is an integer) subgroups of maximal class. Then $c\left(T_{i}\right)$ is odd for $i \in[1,4 t]$ and $c\left(T_{j}\right)$ is even for $j \in[4 t+1, m]$. In this case, by $(1)$, we have $|\mathfrak{M}| \equiv 0(\bmod p)$. If all the $T_{i}$ are not 2-groups of maximal class then by induction $c\left(T_{i}\right) \equiv 0(\bmod p)$ for all $i$, and $|\mathfrak{M}| \equiv 0$ $(\bmod p)$ by $(1)$.

There is a little hope to find $|\mathfrak{M}|\left(\bmod p^{2}\right)$. 
Proof of Theorem A. Induct on $|G|$.

(i) Let $a=e$. Our result is known if $k=1$ (see $\S 1)$. Let $k>1$. We may assume that $\exp G>p^{k}$ (since in the contrary case $N(e, G, k)=|G| \geq p^{k+1}$ ). By supposition and Roquette's theorem (see proof of Lemma 4) $G$ contains a normal subgroup $R$ of type $(p, p)$. Suppose that $G / R$ is cyclic. Then $C_{G}(R)$ is abelian, and its index in $G$ is at most $p$. If $C_{G}(R)=G$ the result is obvious. Let $C_{G}(R)<G$. Take an element $x \in G$ with $|\langle x\rangle| \leq p^{k}$. Consider the subgroup $T=R\langle x\rangle$. Obviously $\exp T \leq p^{k}<\exp G \Rightarrow T<G \Rightarrow T \leq C_{G}(R)$. Hence any element of $G$ of order not exceeding $p^{k}$ is contained in $C_{G}(R)$. So $N(e, G, k)=N\left(e, C_{G}(R), k\right)$. Since $C_{G}(R)$ is abelian, $N\left(e, C_{G}(R), k\right)=$ $p^{k+1}$ or $p^{k+2}$.

So we may assume that $G / R$ is noncyclic. Then $G / R$ contains a normal subgroup $L / R$ such that $G / L$ is abelian of type $(p, p)$. Since $\exp G>p^{k} \geq$ $p^{2}$, it follows that $L>R$. Hence all maximal subgroups of $G$ containing $L$, say $T_{1}, \ldots, T_{p+1}$, are not 2-groups of maximal class (if a 2-group of maximal class contains a normal subgroup of type $(2,2)$ then its order is equal to 8$)$. Since $x^{p} \in L$ for all $x \in G$,

$$
T_{1} \cup \cdots \cup T_{p+1}=G
$$

and

$$
N(e, G, k)=\sum_{i=1}^{p+1} N\left(e, T_{i}, k\right)-p N(e, L, k) .
$$

By Frobenius's theorem of elementary group theory we have $N(e, L, k) \equiv 0$ $\left(\bmod p^{k}\right)$. By induction $N\left(e, T_{i}, k\right) \equiv 0\left(\bmod p^{k+1}\right)$ since all $T_{i}$ are not 2-groups of maximal class. Hence by $(2)$ we have $N(e, G, k) \equiv 0\left(\bmod p^{k+1}\right)$.

(ii) Let $a \neq e$ and let $\mathfrak{M}$ be the set of all cyclic subgroups containing $\langle a\rangle$ of order $p^{k}|\langle a\rangle|$ in $G$. Then by Lemma 1 we have

$$
N(a, G, k)=\sum_{Z \in \mathfrak{M}} N(a, Z, k)=p^{k}|\mathfrak{M}| .
$$

Since $|\mathfrak{M}| \equiv 0(\bmod p)$ by Lemma $4, N(a, G, k) \equiv 0\left(\bmod p^{k+1}\right)$.

Lemma 5. Let $R$ be a normal subgroup of order $p^{p}$ and exponent $p$ in a $p$ group $G ; G / R$ be cyclic of order $p^{m}, m>1 ; T / R$ be a subgroup of index $p$ in $G / R$. Then the nilpotence class of $T$ is at most $p-1$. In particular $T$ is regular.

Proof. Let $K$ be a $G$-admissible subgroup of order $p^{p-2}$ in $R$ (if $p=2$ then $K=1)$. We set $G^{\circ}=G / K, R^{\circ}=R / K, T^{\circ}=T / K$. Then $C_{G^{\circ}}\left(R^{\circ}\right) \geq T^{\circ}$ and $C_{G^{\circ}}\left(R^{\circ}\right)=D / K$ is abelian (see the proof of Theorem A). So the nilpotency class of $D$ is at most $p-1$, and $D$ is regular by a well-known theorem of Hall [8]. Since $D \geq T, T$ is regular also.

Lemma 6. Let $G, R$, and $T$ be as in Lemma 5, $\exp G \geq p^{k}$. Then $N(e, G, k)$ $=p^{k+p-1}$ or $p^{k+p}$ (here $k$ is a positive integer).

Proof. If $\exp G=p^{k}$ then $|G|=p^{k+p-1}$ or $p^{k+p}$ and $N(e, G, k)=|G|$. Hence we may assume that $\exp G>p^{k}$. If $\exp T>p^{k}$ then $T \geq\langle x \in G| x^{p^{k}}=$ $e)$ and the lemma is true since $T$ is regular (Lemma 5). Let $\exp T=p^{k}$. 
Then $\exp G=p^{k+1}$. Let $x$ be an element of $G$ with $|\langle x\rangle| \leq p^{k}$. Suppose that $x \notin T$. Then $G=R\langle x\rangle$ with $R \cap\langle x\rangle=1$. Then $T=R\left\langle x^{p}\right\rangle$ and $\exp T<p^{k}$, a contradiction. Hence all elements of $G$ of order at most $p^{k}$ lie in $T$. Since $T$ is regular, $T=\left\langle x \in G \mid x^{p^{k}}=e\right\rangle$ and $N(e, G, k)=|T|=p^{k+p-1}$ or $p^{k+p}$.

Proof of Theorem B. By Blackburn's theorem [6] any nonexceptional p-group $G$ contains a normal subgroup $R$ of order $p^{p}$ and exponent $p$ (see also [1, 2]). Our result is true if $k=1$ (see [1] or [7]). Now let $k>1$. In virtue of Lemma 6 we may assume that $G / R$ is noncyclic. Hence $G / R$ contains a normal subgroup $L / R$ such that $G / L$ is abelian of type $(p, p)$. Let $T_{1}, \ldots, T_{p+1}$ be all maximal subgroups of $G$ containing $L$. Then, as in the proof of Theorem A, we have

$$
N(e, G, k)=\sum_{i=1}^{p+1} N\left(e, T_{i}, k\right)-p N(e, L, k) .
$$

If $L=R$ then $\exp G=p^{2}=p^{k}, k=2$, and $N(e, G, k)=|G|=p^{p+2}=$ $p^{k+p} \equiv 0\left(\bmod p^{k+p-1}\right)$. So we may assume that $R<L$. Then all $T_{i}$ are nonexceptional. We may assume that $\exp G>p^{k}$. Then $\exp T_{i} \geq p^{k}$ for all $i$, and by induction we have $N\left(e, T_{i}, k\right) \equiv 0\left(\bmod p^{k+p-1}\right)$. We note that $\exp L \geq p^{k}$. If $L$ is of maximal class then $|L|=p^{p+1}$ (since a $p$-group of maximal class and order larger than $p^{p+1}$ does not contain a normal subgroup of or$\operatorname{der} p^{p}$ and exponent $\left.p\right)$. In this case $k=2$ and $N(e, L, k)=p^{p+1}=p^{k+p-1}$ and $N(e, G, k) \equiv 0\left(\bmod p^{k+p-1}\right)$ by $\left(2^{\prime}\right)$. If $L$ is not a group of maximal class then $N(e, L, k) \equiv 0\left(\bmod p^{k+p-1}\right)$ by induction, and again $N(e, G, k)$ $\equiv 0\left(\bmod p^{k+p-1}\right)$.

We note that if $G$ is a nonregular $p$-group of maximal class then $N(e, G, k)$ $\equiv 0\left(\bmod p^{k+p-2}\right)\left(\right.$ here $\left.\exp G \geq p^{k}\right)$. A proof of this result is analogous to the proof of Theorem B. If $G$ is of maximal class, $\exp G \geq p^{k},|G|>p^{p+1}$, then we may prove that $N(e, G, k) \equiv p^{k+p-2}\left(\bmod p^{p+k-1}\right)$ (for $p=2$ see Lemma 2).

Many related results were proved in $[1-5,7]$.

\section{REFERENCES}

1. Ya. G. Berkovich, A generalization of theorems of Ph. Hall and Blackburn and an application to non-regular p-groups, Izv. Akad. Nauk SSSR Ser. Mat. 35 (1971), 800-830. (Russian)

2. __ Finite groups containing at most $p^{p}$ cyclic subgroup of order $p^{n}$, Voprosy Teor Grup $\mathrm{i}$ Gomologic. Alg., Yaroslavl, 2 (1979); Math. Anal. Prilozhen., Rostov-Don, 1981. (Russian)

3. __, On p-groups of finite order, Sibirsk. Mat. Zh. 9 (1968), no. 6, 1284-1306. (Russian)

4. __, On subgroups of finite p-groups, Izv. Vyssh. Uchebn. Zaved. Mat. 2 (1973), 9-17; Math. Anal. Prilozhen., Rostov-Don, t.7, 1975, pp. 108-122. (Russian)

5. __, Subgroup and normal structure of a finite p-group, Dokl. Akad. Nauk SSSR 196 (1971), 255-258. (Russian)

6. N. Blackburn, Generalizations of certain elementary theorems on p-groups, Proc. London Math. Soc. (3) 11 (1961), 1-22.

7. _ Note on a paper of Berkovich, J. Algebra 24 (1973), 323-334.

8. Ph. Hall, A contribution to the theory of groups of prime power order, Proc. London Math. Soc. (3) 36 (1933), 29-95.

9. I. M. Isaacs, Character theory of finite groups, Academic Press, New York, 1976. 
10. A. Kulakoff, Über die Anzahl der eigentlichen Untergruppen und der Elemente von gegebener Ordnung in p-Gruppen, Math. Ann. 104 (1931), 778-793.

11. T. Y. Lam, Artin exponents of finite groups, J. Algebra 9 (1968), 94-119.

12. $\ldots$, On the number of solutions of $x^{p^{k}}=a$ in a p-group, Illinois J. Math. 32 (1988), 575-583.

Department of Mathematics, Afula Research Institute, University of Haifa, 31905 HAIFA, ISRAEL 